\title{
REFLECTION PRINCIPLE FOR SYSTEMS OF FIRST ORDER ELLIPTIC EQUATIONS WITH ANALYTIC COEFFICIENTS $\left({ }^{1}\right)$
}

\author{
BY \\ CHUNG LING YU
}

\begin{abstract}
Let $T$ be a simply connected domain of the $z=x+i y$ plane, whose boundary contains a portion $\sigma$ of the $x$-axis. Also let $A(z, \zeta), B(z, \zeta), F(z, \zeta), \alpha(z)$, $\beta(z)$ and $\rho(z)$ be holomorphic functions for $z, \zeta \in T \cup \sigma \cup \bar{T}$, with $\alpha(z)-i \beta(z) \neq 0$ for $z \in \bar{T} \cup \sigma, \alpha(z)+i \beta(z) \neq 0$ for $z \in T \cup \sigma$. Furthermore, we assume that $\alpha(x)$ and $\beta(x)$ are real valued functions for $x \in \sigma$. Our reflection principle states that for any solution $w=u+i v$ of an equation of the type $\partial w / \partial \bar{z}=A(z, \bar{z}) w+B(z, \bar{z}) \bar{w}+F(z, \bar{z})$ in $T$ under the boundary condition $\alpha(x) u+\beta(x) v=\rho(x)$ on $\sigma, w$ can be continued analytically across the $x$-axis, onto the entire mirror image $\bar{T}$.
\end{abstract}

1. Introduction. The classical reflection principle for the Cauchy-Riemann equations has been generalized by many authors to solutions of various types of elliptic equations with analytic coefficients in the plane. Second order equations have been investigated by Lewy [9], biharmonic equations by Poritsky [11], Duffin [4], Bramble [2], Sloss [12], and polyharmonic equations by Huber [6] and Kraft [7].

Some more general higher order equations have been treated by Brown [3], Sloss [13], and $\mathrm{Yu}[16]$.

Garabedian [5] demonstrated a reflection principle for a very general system of second order quasi-linear elliptic equations, but his results are local and the domains into which reflection is possible are not made explicit, even for linear equations. Kraft [8] has adopted Garabedian's method to reflection principles for $C^{2}$ solutions of a class of first order quasi-linear elliptic system, but again his results are local and not explicit.

Our purpose is to study the reflection principle for elliptic equations of the type

$$
\partial u / \partial x-\partial v / \partial y=a u+b v+f, \quad \partial u / \partial y+\partial v / \partial x=c u+d v+g
$$

Presented to the Society, April 25, 1970; received by the editors March 9, 1970 and, in revised form, December 28, 1970.

AMS 1968 subject classifications. Primary 3544.

Key words and phrases. Reflection principle, first order elliptic equation, pseudo-analytic functions, Cauchy-Riemann equations.

(1) The results presented here are part of the author's doctoral dissertation, which was directed by Professor A. Douglis at the University of Maryland.

This research was supported in part by National Science Foundation grant GP-5424.

Copyright (C) 1972, American Mathematical Society 
where $a, b, c, d, f, g$ are analytic functions of the real variables $x, y$ in a domain of $z=x+i y$ plane. We shall achieve the reflection principle for this by simple explicit formulas which then will give clear information about the domain of extension.

The solutions of equation (M) have many properties similar to those of holomorphic functions. These properties have been studied systematically by Bers and Vekua in their well-known books [1], [14]. But almost nothing was known about the reflection principle.

Our method is to apply Lewy's idea in [9] to the complex representations developed by Vekua [15]. One of the main difficulties encountered in applying the method of Lewy [9] to the case of (M) is that the complex representation (3.11) established by Vekua for the solution $w(z)$ of (2.2) in a domain $G$ cannot immediately be applied to $G \cup \partial G$, where $\partial G$ is the boundary of $G$. The difficulty has been overcome by using the generalized Cauchy integral formula (Theorem 4.2).

In $\$ 2$ we give some definitions and state the main theorem. In $\$ 3$ we follow the method of Vekua [15] to give integral representations of the solutions of $(M)$ in the domain $G$. In $\$ 4$ we extend the integral representation (4.7) to the boundary of the domain on which the solution is given. Then in $\$ 6$ we prove the main theorem.

2. Definitions and the main theorem. We now give a few definitions and properties of holomorphic functions of several complex variables which will be needed later.

Let $f\left(z_{1}, \ldots, z_{n}\right)$ be a holomorphic function of the complex variables $z_{1}, \ldots, z_{n}$ in a domain $G \subset C^{n}$. We can associate with $f\left(z_{1}, \ldots, z_{n}\right)$ another function, defined in the conjugate domain $\bar{G}=\left\{\left(\zeta_{1}, \ldots, \zeta_{n}\right) ;\left(\zeta_{1}, \ldots, \zeta_{n}\right) \in C^{n},\left(\zeta_{1}, \ldots, \zeta_{n}\right) \in G\right\}$ by the formula

$$
f^{*}\left(\zeta_{1}, \ldots, \zeta_{n}\right)=\overline{f\left(\zeta_{1}, \ldots, \zeta_{n}\right)}, \quad\left(\zeta_{1}, \ldots, \zeta_{n}\right) \in \bar{G}
$$

We call $f^{*}\left(\zeta_{1}, \ldots, \zeta_{n}\right)$ the ${ }^{*}$ conjugate function to $f\left(z_{1}, \ldots, z_{n}\right)$. It is easily seen that $f^{*}\left(\zeta_{1}, \ldots, \zeta_{n}\right)$ is a holomorphic function of $\zeta_{1}, \ldots, \zeta_{n}$ in $\bar{G}$.

Now let $g\left(x_{1}, y_{1} ; \ldots ; x_{n}, y_{n}\right)$ be a given analytic function of the real variables $x_{1}, y_{1}, \ldots, x_{n}, y_{n}$, in a domain $G \in C^{n}$. According to the theory of functions of several complex variables there corresponds a function, called the analytic continuation of $g\left(x_{1}, y_{1} ; \ldots ; x_{n}, y_{n}\right)$, which is complex analytic in a domain $H \in C^{2 n}$ of the $2 n$ complex variables $x_{1}, y_{1} ; \ldots ; x_{n}, y_{n}$, containing $G$, and which coincides with $g\left(x_{1}, y_{1} ; \ldots ; x_{n}, y_{n}\right)$ for $\left(x_{1}, y_{1} ; \ldots ; x_{n}, y_{n}\right) \in G$. We shall denote the analytic continuation of $g\left(x_{1}, y_{1} ; \ldots ; x_{n}, y_{n}\right)$ again by $g\left(x_{1}, y_{1} ; \ldots ; x_{n}, y_{n}\right)$.

Let us now introduce $2 n$ new variables $z_{k}, \zeta_{k}(k=1, \ldots, n)$ by the relations

$$
z_{k}=x_{k}+i y_{k}, \quad \zeta_{k}=x_{k}-i y_{k}
$$

the variables $z_{k}$ and $\zeta_{k}$ are conjugate if and only if $x_{k}$ and $y_{k}$ are real. We put

$$
G\left(z_{1}, \zeta_{1}, \ldots, z_{n}, \zeta_{n}\right)=g\left(x_{1}, y_{1}, \ldots, x_{n}, y_{n}\right)
$$

According to the above, $G\left(z_{1}, \zeta_{1}, \ldots, z_{n}, \zeta_{n}\right)$ is holomorphic in $2 n$ complex variables $z_{1}, \zeta_{1}, \ldots, z_{n}, \zeta_{n}$. 
By introducing the complex notation $\partial / \partial \bar{z}=\frac{1}{2}(\partial / \partial x+i \partial / \partial y)$, we can write (M) in the complex form

$$
\partial w / \partial \bar{z}=A w+B \bar{w}+F
$$

where

$$
\begin{aligned}
w & =u+i v, & A & =\frac{1}{4}(a+d+i c-i b), \\
B & =\frac{1}{4}(a-d+i c+i b), & F & =\frac{1}{2}(f+g) .
\end{aligned}
$$

If we continue $a, b, c, d, f, g$ into the complex, we obtain $A, B, F$ as holomorphic functions of the two complex variables $z=x+i y, \zeta=x-i y$.

Throughout this paper we will assume $T$ to be a simply connected domain on the $z$ plane whose boundary $\partial T$ is supposed to contain a segment $\sigma, \sigma=\{x: a<x<b\}$. We assume $\sigma$ to contain the origin as an interior point.

We also assume $A(z, \zeta), B(z, \zeta), F(z, \zeta)$ are holomorphic functions of $z, \zeta \in T$ $\cup \sigma \cup \bar{T}$, where $\bar{T}$ is the conjugate domain of $T$.

The main result in this paper will be Theorem 2.1 .

THEOREM 2.1. Let $w(z)$ be a $C^{\prime}$ solution of differential equation (2.2) in $T$, continuous in $T \cup \sigma$, satisfying

$$
\operatorname{Re}[\overline{\lambda(x)} w] \equiv \alpha u+\beta v=\rho(x), \quad \lambda(x)=\alpha(x)+i \beta(x),
$$

on $\sigma$, where $\rho(z), \alpha(z), \beta(z)$ are holomorphic functions in the domain $T \cup \sigma \cup \bar{T}$, such that $\alpha(z)-i \beta(z) \neq 0$ for $z \in \bar{T} \cup \sigma, \alpha(z)+i \beta(z) \neq 0$ for $z \in T \cup \sigma$. Then $w(z)$ can be continued analytically into the domain $T \cup \sigma \cup \bar{T}$; that is, there exists a unique $w(z)$ which is a $C^{\prime}$ solution of differential equation (2.2) in $T \cup \sigma \cup \bar{T}$ and which agrees with the given $w(z)$ in $T \cup \sigma$.

3. Integral representations and the generalized Cauchy integral formula. In this section we give a brief summary of the representation formulas of the solutions of equation (2.2). These are studied in Vekua [15].

Let $G \subset T \cup \sigma \cup \bar{T}$ be a simply connected domain in the complex plane. Then we have

THEOREM 3.1 (VEKUA [15, p. 82]). Every $C^{\prime}$ solution $w(z)$ of equation (2.2) in $G$ has an analytic continuation $W(z, \zeta)$ to $(G, \bar{G})$ in the complex variables $(z, \zeta)$; that is, $W(z, \zeta)$ is holomorphic for $z \in G, \zeta \in \bar{G}$, and $W(z, \bar{z})=w(z)$ for $z \in G$.

For this continuation,

$$
\partial W / \partial \zeta=A(z, \zeta) W(z, \zeta)+B(z, \zeta) W^{*}(\zeta, z)+F(z, \zeta)
$$

where $W^{*}(\zeta, z)$ is the * conjugate function of $W(z, \zeta)$, and is defined by (2.1). For $\zeta=\bar{z}, \partial W / \partial \zeta=\partial W / \partial \bar{z}$, and (3.1) reduces to (2.2). Moreover, if $W(z, \zeta)$ is a holomorphic function of $z, \zeta$ for $z \in G, \zeta \in \bar{G}$, satisfying (3.1), then $W(z, \bar{z})$ satisfies equation (2.2) in $G$. 
A convenient technical simplification results from using the transformation

$$
W(z, \zeta)=W_{0}(z, \zeta) \exp \int_{\zeta_{1}}^{\zeta} A(z, t) d t
$$

where $\zeta_{1}$ is a fixed point in $\bar{T}$. Substituting (3.2) in (3.1), we obtain the differential equation

$$
\partial W_{0} / \partial \zeta=C(z, \zeta) W_{0}^{*}(\zeta, z)+F_{0}(z, \zeta)
$$

where

$$
\begin{aligned}
& C(z, \zeta)=B(z, \zeta) \exp \left[\int_{\bar{\zeta}_{1}}^{z} A^{*}(\zeta, t) d t-\int_{\zeta_{1}}^{\zeta} A(z, t) d t\right] \\
& F_{0}(z, \zeta)=F(z, \zeta) \exp \left[-\int_{\zeta_{1}}^{\zeta} A(z, t) d t\right] .
\end{aligned}
$$

LEMMA 3.1. Every solution $W_{0}(z, \zeta)$ of equation (3.3), holomorphic in the domain $(G, \bar{G})$, has the following integral representation formula:

$$
\begin{aligned}
W_{0}(z, \zeta)= & \phi(z)+\int_{z_{0}}^{z} \Gamma_{1}\left(z, \zeta, t, \zeta_{0}\right) \phi(t) d t \\
& +\int_{\zeta_{0}}^{\zeta} \Gamma_{2}\left(z, \zeta, z_{0}, \tau\right) \phi^{*}(\tau) d \tau+U_{0}(z, \zeta)
\end{aligned}
$$

where $\left(z_{0}, \zeta_{0}\right)$ is a fixed point in $(G, \bar{G}), \phi(z)$ is a holomorphic function in $G, U_{0}(z, \zeta)$ is a holomorphic function for $z, \zeta \in T \cup \sigma \cup \bar{T}$, and $\Gamma_{1}(z, \zeta, t, \tau), \Gamma_{2}(z, \zeta, t, \tau)$ are two holomorphic functions of the four variables $z, t, \zeta, \tau \in T \cup \sigma \cup \bar{T}$. Furthermore,

$$
\begin{aligned}
\dot{U}_{0}(z, \zeta)= & \int_{\zeta_{0}}^{\zeta} F_{0}(z, \tau) d \tau+\int_{\zeta_{0}}^{\zeta} d \tau \int_{z_{0}}^{z} \Gamma_{1}(z, \zeta, t, \tau) F_{0}(t, \tau) d t \\
& +\int_{\zeta_{0}}^{\zeta} d \tau \int_{z_{0}}^{z} \Gamma_{2}(z, \zeta, t, \tau) F_{0}^{*}(\tau, t) d t
\end{aligned}
$$

and

$$
\begin{gathered}
\Gamma_{1}(z, \zeta, t, \tau)=\int_{\tau}^{\zeta} \Gamma(z, \zeta, t, \eta) d \eta \\
\Gamma_{2}(z, \zeta, t, \tau)=C(z, \tau)+\int_{t}^{z} C(\xi, \tau) \Gamma_{1}(z, \zeta, \xi, \tau) d \xi
\end{gathered}
$$

where $\Gamma$ is the unique holomorphic solution of the Volterra integral equation

$$
\Gamma(z, \zeta, t, \tau)=C(z, \tau) C^{*}(\tau, t)+\int_{\tau}^{\zeta} d \eta \int_{t}^{z} C(\xi, \tau) C^{*}(\tau, t) \Gamma(z, \zeta, \xi, \eta) d \xi
$$

for $z, \zeta, t, \tau \in T \cup \sigma \cup \bar{T}$.

Conversely, if $W_{0}(z, \zeta)$ is given by (3.4) in the domain $(G, \bar{G})$, then $W_{0}(z, \zeta)$ satisfies (3.3) in $(G, \bar{G})$. 
Let $w_{0}(z)=W_{0}(z, \bar{z})$. Then

$$
\partial w_{0} / \partial \bar{z}=C(z, \bar{z}) \overline{w_{0}(z)}+F_{0}(z, \bar{z}) .
$$

By Theorem 3.1 and Lemma 3.1, we have

LEMMA 3.2. Every $C^{\prime}$ solution $w_{0}(z)$ of differential equation (3.9) in $G$ has the integral representation

$$
\begin{aligned}
w_{0}(z)= & \phi(z)+\int_{z_{0}}^{z} \Gamma_{1}\left(z, \bar{z}, t, \zeta_{0}\right) \phi(t) d t \\
& +\int_{\zeta_{0}}^{\bar{z}} \Gamma_{2}\left(z, \bar{z}, z_{0}, \tau\right) \phi^{*}(\tau) d \tau+U_{0}(z, \bar{z}) .
\end{aligned}
$$

Conversely, the function $w_{0}(z)$ which is given by (3.10) is a solution of differential equation (3.9) in $G$.

By Theorem 3.1, Lemma 3.2 and the transformation (3.2), we have

THEOREM 3.2. Every $C^{\prime}$ solution $w(z)$ of differential equation (2.2) in $G$ has the integral representation

$$
\begin{aligned}
w(z)=\{\phi(z)+ & \int_{z_{0}}^{z} \Gamma_{1}\left(z, \bar{z}, t, \zeta_{0}\right) \phi(t) d t \\
& \left.+\int_{\zeta_{0}}^{\bar{z}} \Gamma_{2}\left(z, \bar{z}, z_{0}, \tau\right) \phi^{*}(\tau) d \tau+U_{0}(z, \bar{z})\right\} \exp \int_{\zeta_{1}}^{\bar{z}} A(z, t) d t .
\end{aligned}
$$

Conversely, the function w(z) which is given by formula (3.11) is a solution of differential equation (2.2) in $G$.

Now we are in a position to state the generalized Cauchy integral formula of the homogeneous differential equation

$$
\partial w / \partial \bar{z}=C(z, \bar{z}) \overline{w(z)}
$$

THEOREM 3.3. Let $H$ be a simply connected domain with piecewise smooth boundary $\partial H$ such that $H \cup \partial H \subset T \cup \sigma \cup \bar{T}$, then for any $C^{\prime}$ solution $w(z)$ of differential equation (3.12), continuous in $H \cup \partial H$, we have

$$
w(z)=\frac{1}{2 \pi i} \int_{\partial H}[w(t) U(t, \bar{t}, z, \bar{z}) d t-\overline{w(t)} \overline{V(t, \bar{t}, z, \bar{z})} d \bar{t}]
$$

where

$$
\begin{aligned}
U(t, \bar{t}, z, \bar{z}) & =U_{1}(t, \bar{t}, z, \bar{z})-U_{2}(t, \bar{t}, z, \bar{z}), \\
V(t, \bar{t}, z, \bar{z}) & =V_{1}(t, \bar{t}, z, \bar{z})-V_{2}(t, \bar{t}, z, \bar{z}), \\
U_{1}(t, \bar{t}, z, \bar{z}) & =1 /(t-z)-\Gamma_{1}(z, \bar{z}, t, \bar{t}) \log [(t-z)(\bar{t}-\bar{z})]+\Sigma_{1}, \\
V_{1}(t, \bar{t}, z, \bar{z}) & =-\Gamma_{2}^{*}(\bar{z}, z, \bar{t}, t) \log [(t-z)(\bar{t}-\bar{z})]+\Sigma_{2},
\end{aligned}
$$


and $\Sigma_{1}(t, \eta, z, \zeta), \Sigma_{2}(t, \eta, z, \zeta), U_{2}(t, \eta, z, \zeta), V_{2}(t, \eta, z, \zeta)$ are certain holomorphic functions of $t, \eta, z, \zeta \in T \cup \sigma \cup \bar{T}$, given explicitly in Vekua [15, pp. 77-81].

4. The complex integral representation in $D \cup \sigma_{0}$. One of the main difficulties encountered in proving Theorem 2.1 is that the complex integral representation (3.11) cannot immediately be applied to the boundary of $G$. The following Theorem 4.2 is going to overcome this difficulty.

We shall establish a few lemmas to help us to prove Theorem 4.1 and Theorem 4.2. From now on we will assume $D$ to be a simply connected domain with a smooth boundary $\partial D$ such that $D \cup \partial D \subset T \cup \sigma$. The boundary $\partial D$ is supposed to contain a segment $\sigma_{0}, \sigma_{0}=\left\{x: a<a_{0}<x<b_{0}<b, a_{0}<0, b_{0}>0\right\}$.

Let $w(z)$ be a $C^{\prime}$ function in $D$, Hölder continuous in $D \cup \partial D$. Define the functions

$$
\begin{array}{ll}
g_{1}(z)=\frac{1}{2 \pi i} \int_{\partial D} \frac{w(t)}{t-z} d t, \quad z \in D, & \\
g_{2}(z, \zeta)=\frac{1}{2 \pi i} \int_{\partial D} w(t) \Gamma_{1}(z, \zeta, t, \bar{t}) \log [(t-z)(\bar{t}-\zeta)] d t, & z \in D, \zeta \in \bar{D} \\
g_{3}(z, \zeta)=\frac{1}{2 \pi i} \int_{\partial D} \overline{w(t)} \Gamma_{2}(z, \zeta, t, \bar{t}) \log [(t-z)(\bar{t}-\zeta)] d \bar{t}, & z \in D, \zeta \in \bar{D}, \\
g_{4}(z, \zeta)=\frac{1}{2 \pi i} \int_{\partial D} w(t)\left[\Sigma_{1}(t, \bar{t}, z, \zeta)-U_{2}(t, \bar{t}, z, \zeta)\right] d t, & z \in D, \zeta \in \bar{D}, \\
g_{5}(z, \zeta)=\frac{1}{2 \pi i} \int_{\partial D} \overline{w(t)}\left[\Sigma_{2}^{*}(\bar{t}, t, \zeta, z)-V_{2}^{*}(\bar{t}, t, \zeta, z)\right] d \bar{t}, & z \in D, \zeta \in \bar{D}
\end{array}
$$

where the functions $\Gamma_{1}, \Gamma_{2}, U_{2}, V_{2}^{*}, \Sigma_{1}, \Sigma_{2}$ are defined in the previous section. Recall they are holomorphic functions of $t, \eta, z, \zeta$ for $t, \eta, z, \zeta \in T \cup \sigma \cup \bar{T}$. We have

LEMMA 4.1. The function $g_{1}(z)$ is holomorphic in $D$, and can be defined as a Hölder continuous function in $D \cup \partial D$.

Proof. See Mushelišvili [10].

LEMMA 4.2. The function $g_{2}(z, \zeta)$ is holomorphic in $(D, \bar{D})$ and for a fixed point $\zeta_{0} \in \bar{D}, g_{2}\left(z, \zeta_{0}\right)$ is continuous in $D \cup \sigma_{0}, \sigma_{0}=\left\{x: a_{0}<x<b_{0}\right\}$.

Proof. If $t \in \partial D$, the function $\log [(t-z)(\bar{t}-\zeta)]$ of $(z, \zeta)$ is single-valued and holomorphic w.r.t. $z, \zeta$ for $(z, \zeta) \in(D, \bar{D})$. Hence the same is true for

$$
w(t) \Gamma_{1}(z, \zeta, t, \bar{t}) \log [(t-z)(\bar{t}-\zeta)] .
$$

Since integration with respect to $t$ preserves this property, $g_{2}(z, \zeta)$ is a holomorphic function for $z \in D, \zeta \in \bar{D}$ as asserted. 
We write

$$
\begin{aligned}
g_{2}(z, \zeta)= & \frac{1}{2 \pi i} \int_{\partial D} w(t) \Gamma_{1}(z, \zeta, t, \bar{t}) \log [(t-z)(\bar{t}-\zeta)] d t \\
= & \frac{1}{2 \pi i} \int_{\partial D-\sigma_{0}} w(t) \Gamma_{1}(z, \zeta, t, \bar{t}) \log [(t-z)(\bar{t}-\zeta)] d t \\
& +\frac{1}{2 \pi i} \int_{a_{0}}^{b_{0}} w(t) \Gamma_{1}(z, \zeta, t, t) \log (t-z) d t \\
& +\frac{1}{2 \pi i} \int_{a_{0}}^{b_{0}} w(t) \Gamma_{1}(z, \zeta, t, t) \log (t-\zeta) d t \\
= & \mathrm{I}(z, \zeta)+\operatorname{II}(z, \zeta)+\operatorname{III}(z, \zeta) .
\end{aligned}
$$

Let $D_{1}$ be a subdomain of $D$, the boundary $\partial D_{1}$ of which contains a segment $\sigma_{1}$, where $\sigma_{1}$ is a closed subinterval of $\sigma_{0}$. Furthermore, assume that the distance from $D_{1}$ to $\partial D \backslash \sigma_{0}$ is greater than zero. Thus, for $t \in \partial D \mid \sigma_{0}, z \in D_{1} \cup \sigma_{1}$, the function

$$
w(t) \Gamma_{1}\left(z, \zeta_{0}, t, \bar{t}\right) \log \left[(t-z)\left(\bar{t}-\zeta_{0}\right)\right]
$$

is uniformly continuous. Therefore $\mathrm{I}\left(z, \zeta_{0}\right)$ is continuous for $z$ on $D \cup \sigma_{0}$.

Using integration by parts, we obtain for $a_{0} \leqq x \leqq b_{0}$,

$$
\begin{aligned}
\mathrm{II}\left(z, \zeta_{0}\right)= & \frac{1}{2 \pi i}\left\{w(x) \Gamma_{1}\left(z, \zeta_{0}, x, x\right)(x-z) \log (x-z)-w(x) \Gamma_{1}\left(z, \zeta_{0}, x, x\right)(x-z)\right\}_{x=a_{0}}^{x=b_{0}} \\
& -\frac{1}{2 \pi i} \int_{a_{0}}^{b_{0}}\{(x-z) \log (x-z)-(x-z)\} \frac{d}{d x}\left\{w(x) \Gamma_{1}\left(z, \zeta_{0}, x, x\right)\right\} d x .
\end{aligned}
$$

Since if $x \in \sigma_{0}$ the function $(x-z) \log (x-z)-(x-z)$ is continuous for any $z \in D \cup \sigma_{0}$, it follows that $\operatorname{II}\left(z, \zeta_{0}\right)$ is continuous in $D \cup \sigma_{0}$.

A similar integration by parts proves that $\operatorname{III}\left(z, \zeta_{0}\right)$ is continuous in $D \cup \sigma_{0}$. This completes the proof.

Using the same method, we get the following

LEMMA 4.3. The function $g_{3}(z, \zeta)$ is holomorphic in $(D, \bar{D})$, and for fixed $\zeta_{0} \in \bar{D}$, $g_{3}\left(z, \zeta_{0}\right)$ is continuous in $D \cup \sigma_{0}, \sigma_{0}=\left\{x: a_{0}<x<b_{0}\right\}$.

LEMMA 4.4. The functions $g_{4}(z, \zeta), g_{5}(z, \zeta)$ are holomorphic in $(D, \bar{D})$ and for fixed $\zeta_{0} \in \bar{D}, g_{4}\left(z, \zeta_{0}\right)$ and $g_{5}\left(z, \zeta_{0}\right)$ are continuous in $D \cup \sigma_{0} \cup \bar{D}$.

Proof. This follows since the integrands are holomorphic functions of $z, \zeta \in \bar{T}$ $\cup \sigma \cup \bar{T}$.

THEOREM 4.1. Let $w_{0}(z)$ be a $C^{\prime}$ solution of differential equation (3.9) in $D$, Hölder continuous in $D \cup \partial D$. Then there is a holomorphic function $W_{0}(z, \zeta), z \in D, \zeta \in \bar{D}$, such that $w_{0}(z)=W_{0}(z, \bar{z})$. Furthermore, for a fixed point $\zeta_{0} \in \bar{D}, W_{0}\left(z, \zeta_{0}\right)$ is holomorphic in $D$ and continuous in $D \cup \sigma_{0}$. 
Proof. By Lemma 3.2 and (3.5), there exists a solution $w_{1}(z)$ of homogeneous differential equation (3.12) in $D$ such that

$$
w_{0}(z)=w_{1}(z)+U_{0}(z, \bar{z}) .
$$

Since $U_{0}(z, \zeta)$ is a holomorphic function for $z, \zeta \in T \cup \sigma \cup \bar{T}$, therefore, $w_{1}(z)$ is Hölder continuous in $D \cup \partial D$. Define

$$
W_{1}(z, \zeta)=\frac{1}{2 \pi i} \int_{\partial D}\left[w_{1}(t) U(t, \bar{t}, z, \zeta) d t-\overline{w_{1}(t)} V^{*}(\bar{t}, t, \zeta, z) d \bar{t}\right],
$$

where $U(t, \tau, z, \zeta), V(t, \tau, z, \zeta)$ are given by Theorem 3.3. It is easy to see that

$$
W_{1}(z, \zeta)=g_{1}(z)-g_{2}(z, \zeta)+g_{3}(z, \zeta)+g_{4}(z, \zeta)-g_{5}(z, \zeta),
$$

where $g_{1}, g_{2}, g_{3}, g_{4}, g_{5}$ are defined by (4.1), (4.2), (4.3), (4.4), (4.5) respectively. By Lemmas 4.1 through 4.4 , the function $W_{1}(z, \zeta)$ is defined as a holomorphic function for $z \in D, \zeta \in \bar{D}$, and for a fixed point $\zeta_{0} \in \bar{D}, W_{1}\left(z, \zeta_{0}\right)$ is holomorphic in $D$ and continuous in $D \cup \sigma_{0}$. By Theorem 3.3, we have $W_{1}(z, \bar{z})=w_{1}(z)$ in $D$, so the function $W_{0}(z, \zeta)=W_{1}(z, \zeta)+U(z, \zeta)$ serves the purpose; this completes the proof.

THEOREM 4.2. Let $w(z)$ be a $C^{\prime}$ solution of differential equation (2.2) in D, Hölder continuous on $D \cup \partial D$. Then

$$
\begin{aligned}
w(z)=\left\{\phi(z)+\int_{z_{0}}^{z} \Gamma_{1}\left(z, \bar{z}, t, \bar{z}_{0}\right) \phi(t)\right. \\
\left.\quad+\int_{\bar{z}_{0}}^{\bar{z}} \Gamma_{2}\left(z, \bar{z}, z_{0}, \tau\right) \phi^{*}(\tau) d \tau+U_{0}(z, \bar{z})\right\} \exp \int_{\zeta_{1}}^{\bar{z}} A(z, t) d t,
\end{aligned}
$$

where $\phi(z)$ is a function holomorphic in $D$, and can be defined as a continuous function in $D \cup \sigma_{0}$. Conversely, the function $w(z)$ which is given by (4.7) is a solution of differential equation (2.2) in $D$, continuous in $D \cup \sigma_{0}$.

Proof. By Theorem 3.1, we have a holomorphic function $W(z, \zeta), z \in D, \zeta \in \bar{D}$, such that $W(z, \bar{z})=w(z)$. Moreover, (3.2) and Lemma 3.1 give the representation

$$
\begin{aligned}
W(z, \zeta)= & W_{0}(z, \zeta) \exp \left[\int_{\zeta_{1}}^{\zeta} A(z, t) d t\right] \\
= & \left\{\phi(z)+\int_{z_{0}}^{z} \Gamma_{1}\left(z, \zeta, t, \bar{z}_{0}\right) \phi(t) d t\right. \\
& \left.+\int_{\bar{z}_{0}}^{\zeta} \Gamma_{2}\left(z, \zeta, z_{0}, \tau\right) \phi^{*}(\tau) d \tau+U_{0}(z, \zeta)\right\} \exp \left[\int_{\zeta_{1}}^{\zeta} A(z, t) d t\right],
\end{aligned}
$$

where $\phi(z)$ is a function holomorphic in $D$. Next, observe that $w(z)$ is Hölder continuous in $D \cup \partial D$ if and only if $w_{0}(z)$ is Hölder continuous in $D \cup \partial D$, where $w_{0}(z)=W_{0}(z, \bar{z})$. By Theorem 4.1, $W_{0}\left(z, \bar{z}_{0}\right)$ is holomorphic in $D$ and continuous in $D \cup \sigma_{0}$, where $\bar{z}_{0}$ is a fixed point in $\bar{D}$. By (3.6), $W_{0}\left(z, \bar{z}_{0}\right)=\phi(z)+U_{0}\left(z, \bar{z}_{0}\right)$. Thus $\phi(z)$ is holomorphic in $D$ and can be defined as a continuous function in $D \cup \sigma_{0}$. 
5. The complex Volterra integral equations. The complex Volterra integral equations in domain $D$ have been studied in detail in Vekua [15]. The purpose of this section is to study the Volterra integral equation

$$
g(z)=f(z)+\int_{0}^{z} K(z, t) g(t) d t, \quad z \in D \cup \sigma_{0},
$$

where $D$ and $\sigma_{0}$ are defined in $\S 4, f(z)$ is continuous in $D \cup \sigma_{0}$ and holomorphic in $D$, and $K(z, t)$ is holomorphic in $(T \cup \sigma \cup \bar{T}, T \cup \sigma \cup \bar{T})$.

THEOREM 5.1. The integral equation (5.1) has a unique solution $g(z)$ which is continuous in $D \cup \sigma_{0}$ and holomorphic in $D$.

Proof. To prove the existence of a solution, we construct a sequence of functions $g_{n}(z)(n=1,2, \ldots)$ as follows:

$$
g_{0}(z)=f(z), \quad g_{n}(z)=f(z)+\int_{0}^{z} K(z, t) g_{n-1}(t) d t .
$$

It is evident that if

$$
\lim _{n \rightarrow \infty} g_{n}(z)=g(z)
$$

exists uniformly on every compact subset of $D \cup \sigma_{0}$, then $g(z)$ is a solution of (5.1) in $D$.

To demonstrate the uniform convergence, we observe that

$$
g_{n}(z)=f(z)+\int_{0}^{z} \Gamma^{n}(z, t) f(t) d t
$$

where

$$
\Gamma^{n}(z, t)=K^{\prime}(z, t)+\cdots+K^{n}(z, t)
$$

and

$$
K^{\prime}(z, t)=K(z, t), \quad K^{i+1}(z, t)=\int_{t}^{z} K(z, \zeta) K^{i}(\zeta, t) d \zeta
$$

The sum of the right of (5.4) is easily seen to converge uniformly in every compact subset of $(T \cup \sigma \cup \bar{T}, T \cup \sigma \cup \bar{T})$ by the principle of dominated convergence, and this established (5.4).

To prove the uniqueness, let $f(z)=0$, then (5.1) becomes

$$
g(z)=\int_{0}^{z} K(z, t) g(t) d t
$$

Since we assume $g(z)$ is holomorphic in $D$, and continuous in $D \cup \sigma_{0}$, therefore the integration on the right side on (5.5) is independent of the path in $D \cup \sigma_{0}$. 
Let $t=\lambda(\theta), 0 \leqq \theta \leqq 1$, denote the path, for which $0=\lambda(0), z_{0}=\lambda(1)$. Assume $\left|\lambda^{\prime}(\theta)\right| \leqq q, K(z, t) \leqq M$. Hence, if $z$ is a point of the path, $z=\lambda(\beta)$, we have

$$
\begin{aligned}
|g(z)| & =\left|\int_{0}^{z} K(z, t) g(t) d t\right| \leqq M \int_{0}^{z}|g(t)||d t| \\
& =M \int_{0}^{\beta}|g(\lambda(\theta))|\left|\lambda^{\prime}(\theta)\right| d \theta \leqq M q \int_{0}^{\beta}|g(\lambda(\theta))| d \theta .
\end{aligned}
$$

Letting $h(\theta)=|g(\lambda(\theta))|$, we thus have

$$
h(\beta) \leqq M q \int_{0}^{\beta} h(\theta) d \theta=M q A, \quad \text { where } A=\int_{0}^{\beta} h(\theta) d \theta .
$$

Using this inequality again, we have $h(\beta) \leqq A M^{2} q^{2} \beta$. In a similar manner,

$$
h(\beta) \leqq M^{n+1} q^{n+1} A \beta^{n} / n !, \quad n=0,1, \ldots
$$

The last inequality holds for any $n$, and the right side can be made as small as we wish by choosing $n$ sufficiently large. It follows, therefore, that $|g(\lambda(\theta))|=h(\theta)$ $\equiv 0$. This completes the proof.

(A similar estimate will prove that the iteration procedure converges.)

The same method to prove uniqueness can give the following:

COROLlaRY 5.1. The integral equation

$$
w(x)=\int_{0}^{x} K(x, t) w(t) d t, \quad x, t \in \sigma_{0},
$$

has only the trivial solution in $L^{2}\left(a_{0}, b_{0}\right)$.

6. Proof of the main theorem. We first establish the reflection principle in $D \cup \sigma_{0}$, where $D$ and $\sigma_{0}$ are defined in $\S 4$.

LeMmA 6.1. Let $w(z)=u+i v$ be a $C^{\prime}$ solution of differential equation (2.2) in $D$, Hölder continuous in $D \cup \partial D$, satisfying

$$
\operatorname{Re}[\overline{\lambda(x)} w] \equiv \alpha u+\beta v=\rho(x), \quad x \in \sigma_{0}, \lambda=\alpha+i \beta,
$$

where $\rho(z), \alpha(z), \beta(z)$ are holomorphic functions in the domain $D \cup \sigma_{0} \cup \bar{D}$, such that $\alpha(z)-i \beta(z) \neq 0$ for $z \in \bar{D} \cup \sigma_{0}, \alpha(z)+i \beta(z) \neq 0$ for $z \in D \cup \sigma_{0}$. Then $w(z)$ can be continued analytically into the domain $D \cup \sigma_{0} \cup \bar{D}$, i.e. there exists a unique $w(z)$ which is a $C^{\prime}$ solution of differential equation (2.2) in $D \cup \sigma_{0} \cup \bar{D}$ and which agree with the given $w(z)$ in $D \cup \sigma_{0}$.

Proof. By Theorem 4.2, we have the integral representation

$$
\begin{aligned}
w(z)=\{ & \phi(z)+\int_{z_{0}}^{z} \Gamma_{1}\left(z, \bar{z}, t, \bar{z}_{0}\right) \phi(t) d t \\
& \left.\quad+\int_{z_{0}}^{\bar{z}} \Gamma_{2}\left(z, \bar{z}, z_{0}, \tau\right) \phi^{*}(\tau) d \tau+U_{0}(z, \bar{z})\right\} \exp \left[\int_{\zeta_{1}}^{\bar{z}} A(z, t) d t\right],
\end{aligned}
$$


where $\phi(z)$ is a function holomorphic in $D$, and can be defined as a continuous function in $D \cup \sigma_{0}$. Thus

$$
\begin{aligned}
\overline{w(z)}=\left\{\phi^{*}(\bar{z})+\int_{\bar{z}_{0}}^{\bar{z}} \Gamma_{1}^{*}\left(\bar{z}, z, t, z_{0}\right) \phi^{*}(t) d t\right. \\
\left.+\int_{z_{0}}^{z} \Gamma_{2}^{*}\left(\bar{z}, z, \bar{z}_{0}, \tau\right) \phi(\tau) d \tau+U_{0}^{*}(\bar{z}, z)\right\} \exp \left[\int_{\bar{\zeta}_{1}}^{z} A^{*}(\bar{z}, t) d t\right] .
\end{aligned}
$$

Condition (6.1) implies

$$
2 \rho(x)=(\alpha(x)-i \beta(x)) w(x)+(\alpha(x)+i \beta(x)) \overline{w(x)}, \quad x \in \sigma_{0} .
$$

Set

$$
\begin{aligned}
& G_{1}(z)=(\alpha(z)-i \beta(z)) \exp \left[\int_{\zeta_{1}}^{z} A(z, t) d t\right] \\
& G_{2}(z)=(\alpha(z)+i \beta(z)) \exp \left[\int_{\bar{\zeta}_{1}}^{z} A^{*}(z, t) d t\right]
\end{aligned}
$$

then $G_{1}(z) \neq 0$ for $z \in \bar{D} \cup \sigma_{0}$ and $G_{2}(z) \neq 0$ for $z \in D \cup \sigma_{0}$. Substituting (6.2) and (6.3) into (6.4), we obtain the relation for $z \in \sigma_{0}$ (i.e. for $x \in \sigma_{0}$ ),

$$
\begin{aligned}
2 \rho(z)= & G_{1}(z) \phi(z)+G_{2}(z) \phi^{*}(z) \\
& +\int_{z_{0}}^{z}\left\{\Gamma_{1}\left(z, z, t, \bar{z}_{0}\right) G_{1}(z)+\Gamma_{2}^{*}\left(z, z, \bar{z}_{0}, t\right) G_{2}(z)\right\} \phi(t) d t \\
& +\int_{\bar{z}_{0}}^{z}\left\{\Gamma_{2}\left(z, z, \dot{z}_{0}, t\right) G_{1}(z)+\Gamma_{1}^{*}\left(z, z, t, z_{0}\right) G_{2}(z)\right\} \phi^{*}(t) d t \\
& +G_{1}(z) U_{0}(z, z)+G_{2}(z) U_{0}^{*}(z, z) .
\end{aligned}
$$

Recall that $\Gamma_{1}\left(z, z, t, \bar{z}_{0}\right), \Gamma_{2}\left(z, z, z_{0}, \tau\right), \Gamma_{1}^{*}\left(z, z, t, z_{0}\right), \Gamma_{2}^{*}\left(z, z, \bar{z}_{0}, \tau\right), U_{0}(z, z)$, $U_{0}^{*}(z, z), G_{1}(z), G_{2}(z)$ and $\rho(z)$ are holomorphic for $z, t, \tau \in D \cup \sigma_{0} \cup \bar{D}$. We also see that $\phi^{*}(z)$ is known for $z \in \bar{D} \cup \sigma_{0}$. Then (6.5) suggests a Volterra integral equation for an unknown function $\psi(z)$ in $\bar{D} \cup \sigma_{0}$, as follows:

$$
f(z)=G_{1}(z) \psi(z)+\int_{0}^{z}\left\{\Gamma_{1}\left(z, z, t, \bar{z}_{0}\right) G_{1}(z)+\Gamma_{2}^{*}\left(z, z, \bar{z}_{0}, t\right) G_{2}(z)\right\} \psi(t) d t
$$

where

$$
\begin{aligned}
f(z)= & 2 \rho(z)-G_{2}(z) \phi^{*}(z) \\
& -\int_{z_{0}}^{0}\left\{\Gamma_{1}\left(z, z, t, \bar{z}_{0}\right) G_{1}(z)+\Gamma_{2}^{*}\left(z, z, \bar{z}_{0}, t\right) G_{2}(z)\right\} \phi(t) d t \\
& -\int_{\bar{z}_{0}}^{z}\left\{\Gamma_{2}\left(z, z, z_{0}, t\right) G_{1}(z)+\Gamma_{1}^{*}\left(z, z, t, z_{0}\right) G_{2}(z)\right\} \phi^{*}(t) d t \\
& -G_{1}(z) U_{0}(z, z)-G_{2}(z) U_{0}^{*}(z, z) .
\end{aligned}
$$


By Theorem 5.1, the solution $\psi(z)$ must exist and be unique in all of $\bar{D} \cup \sigma_{0}$. It must be holomorphic in $\bar{D}$ and continuous in $\bar{D} \cup \sigma_{0}$. But $\phi(z)$ is holomorphic in $D$ and continuous in $D \cup \sigma_{0}$ and satisfies the same equation (6.6) for $z=x$ on $\sigma_{0}$. By uniqueness (Corollary 5.1), we have $\phi(z)=\psi(z)$ on $\sigma_{0}$.

This $\psi(z)$ serves as the analytic continuation of $\phi(z)$ into $D \cup \sigma_{0} \cup \bar{D}$. In analogous manner, we also can construct the analytic continuation of $\phi^{*}(z)$ into $D \cup \sigma_{0} \cup \bar{D}$.

We now can use (6.2) and Theorem 3.2 to obtain $w(z)$ for arbitrary $z \in D \cup \sigma_{0}$ $\cup \bar{D}$ as an analytic continuation of $w(z)$ for $z \in D \cup \sigma_{0}$ as given originally.

The uniqueness follows from the analyticity of the solution of differential equation (2.2). This completes the proof.

The above lemma assumes that $w(z)$ is Hölder continuous for $z \in D \cup \sigma_{0}$. However, in view of the following lemma, we only need assume that $w(z)$ is continuous for $z \in D \cup \sigma_{0}$.

LEMMA 6.2 (VEKUA [14, p. 313]). Let $w(z)$ be a $C^{\prime}$ solution of differential equation (2.2) in $D$, continuous in $D \cup \partial D$, satisfying

$$
\alpha u+\beta v \equiv \operatorname{Re}[\overline{\lambda(z)} w]=\rho(z), \quad \lambda=\alpha+i \beta \text { on } \partial D,
$$

where $\rho(z), \lambda(z)$ are Hölder continuous with index $\mu, 0<\mu<1,|\lambda(z)|=1$ on $\partial D$. Then $w(z)$ is Hölder continuous with index $\mu$ on $D \cup \partial D$.

Now we are in a position to prove the main theorem.

Proof of Theorem 2.1. By Lemma 6.2, it is easy to see that $w(z)$ is Hölder continuous in $D \cup \partial D$. Then by Lemma $6.1 w(z)$ can be continued analytically into the domain $D \cup \sigma_{0} \cup \bar{D}$. But we can take $D$ so that its boundary is as close as desired to the boundary of $T$. This completes the proof.

\section{REFERENCES}

1. L. Bers, Theory of pseudo-analytic functions, Institute for Mathematics and Mechanics, New York University, New York, 1953. MR 15, 211.

2. J. H. Bramble, Continuation of biharmonic functions across circular arcs, J. Math. Mech. 7 (1958), 905-924. MR 20 \#6614.

3. R. D. Brown, Reflection laws of fourth order elliptic differential equations in two independent variables, J. Math. Mech. 13 (1964), 365-383. MR 29 \#2526.

4. R. J. Duffin, Continuation of biharmonic functions by reflection, Duke Math. J. 22 (1955), 313-324. MR 18, 29.

5. P. R. Garabedian, Analyticity and reflection for plane elliptic systems, Comm. Pure Appl. Math. 14 (1961), 315-322. MR 25 \#309.

6. A. Huber, On the reflection principle for polyharmonic functions, Comm. Pure Appl. Math. 9 (1956), 471-478. MR 19, 26.

7. R. Kraft, Reflection of polyharmonic functions in two independent variables, J. Math. Anal. Appl. 19 (1967), 505-518. MR 35 \#4458.

8. - Analyticity and reflectivity for first order systems of elliptic type in two independent variables, J. Math. Anal. Appl. 29 (1970), 1-17. 
9. H. Lewy, On the reflection laws of second order differential equations in two independent variables, Bull. Amer. Math. Soc. 65 (1959), 37-58. MR 21 \#2810.

10. N. I. Mushelišvili, Singular integral equations. Boundary problems of function theory and their application to mathematical physics, OGIZ, Moscow, 1946; English transl., Noordhoff, Groningen, 1953. MR 8, 586; MR 15, 434.

11. H. Poritsky, On reflection of singularities of harmonic functions corresponding to the boundary condition $\alpha u / \alpha n+a u=0$, Bull. Amer. Math. Soc. 43 (1937), 873-884.

12. J. M. Sloss, Reflection of biharmonic functions across analytic boundary conditions with examples, Pacific J. Math. 13 (1963), 1401-1415. MR 28 \#350.

13. - Reflection laws of high order elliptic differential equations in two independent variables with constant coefficients and unequal characteristics across analytic boundary conditions, Duke Math. J. 35 (1968), 415-434. MR 37 \#4402.

14. I. N. Vekua, Generalized analytic functions, Fizmatgiz, Moscow, 1959; English transl., Pergamon Press, London; Addison-Wesley, Reading, Mass., 1962. MR 21 \#7288; MR 27 \#321.

15. - New methods for solving elliptic equations, OGIZ, Moscow, 1948; English transl., Series in Appl. Math., vol. 1, North-Holland, Amsterdam; Interscience, New York, 1967. MR 11, 598; MR 35 \#3243.

16. C. L. Yu, Reflection principle for solutions of higher order elliptic equations with analytic coefficients, SIAM J. Appl. Math. 20 (1971), 358-363.

Florida State University, Tallahassee, Florida 32306 